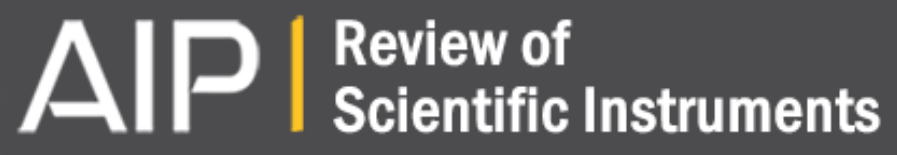

\section{Joule heating scanning structure system}

\section{J. A. Moya}

Citation: Review of Scientific Instruments 87, 085116 (2016); doi: 10.1063/1.4961472

View online: http://dx.doi.org/10.1063/1.4961472

View Table of Contents: http://scitation.aip.org/content/aip/journal/rsi/87/8?ver=pdfcov

Published by the AIP Publishing

\section{Articles you may be interested in}

Magnetic properties of $120-\mathrm{mm}$ wide ribbons of high B s and low core-loss NANOMET® alloy

J. Appl. Phys. 117, 17B715 (2015); 10.1063/1.4913936

Induced anisotropy in FeCo-based nanocomposites: Early transition metal content dependence

J. Appl. Phys. 115, 17A335 (2014); 10.1063/1.4867124

Low temperature magnetic behaviour of glass-covered magnetic microwires with gradient nanocrystalline microstructure

J. Appl. Phys. 115, 033903 (2014); 10.1063/1.4862540

Effect of $\mathrm{P}$ addition on nanocrystallization and high temperature magnetic properties of low $\mathrm{B}$ and $\mathrm{Nb}$ containing FeCo nanocomposites

J. Appl. Phys. 111, 07A301 (2012); 10.1063/1.3670056

Crystallization behavior and high temperature magnetic phase transitions of $\mathrm{Nb}$-substituted $\mathrm{FeCoSiBCu}$ nanocomposites

Appl. Phys. Lett. 99, 192506 (2011); 10.1063/1.3660245

\section{Welcome to a}

\section{Smarter Search \\ with the redesigned \\ Physics Today Buyer's Guide}

PHYSICS

TODAY

Find the tools you're looking for today! 


\title{
Joule heating scanning structure system
}

\author{
J. A. Moya \\ Grupo Interdisciplinario en Materiales, IESIING, INTECIN UBA-CONICET, Universidad Católica de Salta, \\ Salta, Argentina
}

(Received 18 June 2016; accepted 7 August 2016; published online 25 August 2016)

\begin{abstract}
A method and its system to study the structural evolution on soft magnetic amorphous and nanocrystalline ribbon shaped alloys are presented. With only one sample, a complete set of magnetic and electric data at room and at high temperature are obtained in a relatively short period of time, allowing us to elucidate the structural changes occurring in the alloy and to determine the optimal soft magnetic properties annealing conditions. Published by AIP Publishing. [http://dx.doi.org/10.1063/1.4961472]
\end{abstract}

\section{INTRODUCTION}

Soft magnetic materials are mainly used as core of electric transformers, parts in electric motors, various types of sensors, electromagnetic shielding material, etc. The global market tendencies of these materials rank the amorphous metals with the fastest growing rate perspectives today. ${ }^{1}$ Amorphous metals are materials with disordered non-equilibrium structure which evolve towards various metastable states (with different degrees of structural order) when subjected to heat treatments. This structural evolution produces changes in its magnetic properties and optimal properties for the alloy can be obtained with the requirement of its final application. Nanocrystalline soft magnetic materials are an evolution of their amorphous precursor and represent nowadays an emerging industry. The structure of these materials consists in a fine dispersion of nanograins (between 5 and $20 \mathrm{~nm}$ diameter) embedded in an amorphous matrix. Their magnetic properties depend mainly on the size, chemical composition, and crystalline fraction of the nanograins; three parameters that depend heavily on the chemical composition of the initial amorphous alloy and on the supplied heat treatment. The role of the amorphous remnant matrix will be to provide the magnetic exchange interaction among nanograins and to contribute (or not) to the magnetoelastic energy reduction of the material. In some cases, the magnetic properties can be refined by thermomechanical or thermomagnetic heat treatments. For these reasons, the basic equipment for studying the amorphous and nanocrystalline soft magnetic materials consists of heat treatment furnaces and facilities for the determination of magnetic properties at low and high frequencies (saturation magnetization, coercivity, permeability frequency, etc.).

In this paper, we introduce a scanning structural system and method with the capacity of measuring a series of magnetic and electrical properties of amorphous and nanocrystalline metal ribbons as they are subjected to short joule heating treatments (JH3S). Measurements of these properties over the ribbon are carried out in two situations: when the ribbon is at high temperature and when it is at room temperature during and after the short heat treatment, respectively.

\section{EXPERIMENTAL SETUP}

The device consists of a pair of $10 \mathrm{~cm}$ long inductive coils placed collinearly with each other: one of them for field generation at low frequency (e.g., $50 \mathrm{~Hz}$ ) in order to obtain hysteresis cycles curves with the fluxmeter technique (coil field constant $k=3312 \mathrm{~m}^{-1}$ ), and the other for high frequency (e.g., $100 \mathrm{kHz}$ ) for determining magnetic permeability with the lock-in technique $\left(k=585 \mathrm{~m}^{-1}\right)$. Likewise, a pair of pickup coils (1500 turns and 80 turns for low and high frequency, respectively) are placed separately along a glass tube inside which the amorphous ribbon to be tested is inserted. Air flux compensation was performed digitally. The extremes of the ribbon are clamped to electrical terminals where the current is input in the ribbon for Joule annealing. In this way, it can be possible to perform the heat treatment on the sample while simultaneously measuring the magnetic properties as well as monitoring the electrical resistance. To avoid effects of mechanical stresses in the sample due to the load produced by the lower electrode, this is maintained in neutral weight by making use of a balance beam. A schematic view of the device is shown in Fig. 1, where the optional preference for thermomagnetic heat treatments (Helmholtz coils) and for thermomechanical treatments (stress annealing with mechanical load) is also shown. This configuration requires short annealing times in order not to compromise the integrity of the pick-up coils because of the heat dissipated during the heat treatments.

This first prototype does not measure the treatment temperature of the ribbon during the annealing process. An attempt to determine the temperature while submitting the sample to the heat treatments was performed by a microthermocouple (wire diameter $75 \mu \mathrm{m}$ ) and did not yield good results. However, it is used to determine when the system reaches the room temperature.

The peripheral equipment (see Fig. 2) is composed basically by the ELVIS platform from National Instruments (an acquiring data card that incorporates 12 virtual instruments), a power amplifier (KEPCO BOP $200 \mathrm{~W}$ ) that provides the current for the annealing treatments and a voltage amplifier to power the $H$ field of the hysteresis loops. Current annealing treatments were performed with a typical experimental setup for Joule heating, ${ }^{2}$ in air atmosphere. The sample resistance, 


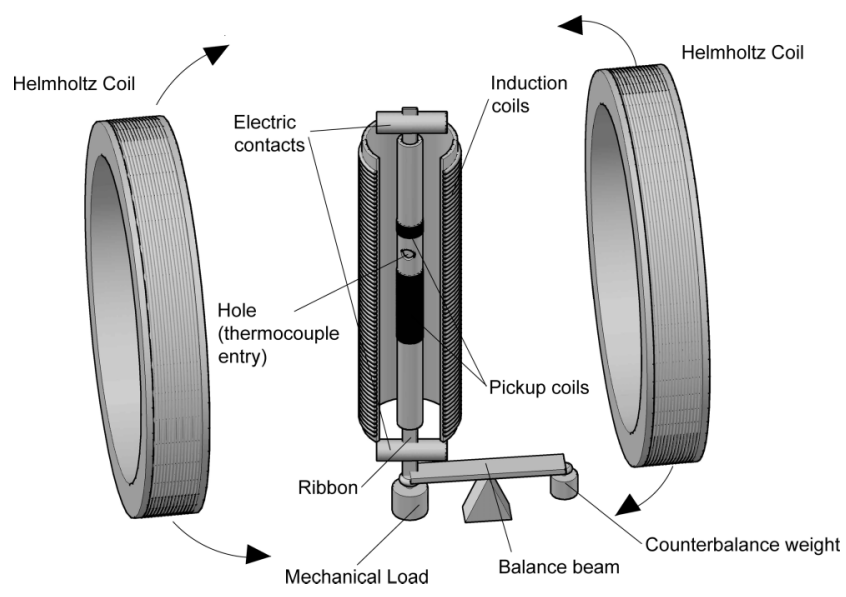

FIG. 1. Schematic view of the JH3S device together with Helmholtz coils and mechanical load optional preferences. Some objects are not in scale.

$R$, was determined by measuring the voltage drop, $V_{\mathrm{r}}$, in a $2 \Omega$ resistor, $R_{\mathrm{r}}$ (connected in series to the ribbon) simultaneously with the voltage drop in the sample, $V_{\mathrm{s}}$, and performing $V_{\mathrm{s}} / R=V_{\mathrm{r}} / R_{\mathrm{r}}$. Hysteresis loop tracer $^{3}$ and permeability (lockin) measurement virtual instruments developed in Labview software were employed for determining the magnetic characteristic of the materials in low and high frequency, respectively. The user interface was also developed in Labview program and controls the Joule annealing treatments (electrical current) and records the sample resistance measurements together with the magnetic measurement virtualized instruments.

\section{EXPERIMENTAL}

As an example of the studies carried out with JH3S system, we employed an amorphous ribbon of the nanocrystalline composition $\mathrm{Fe}_{73.5} \mathrm{Si}_{16.5} \mathrm{~B}_{6} \mathrm{Nb}_{3} \mathrm{Cu}_{1}$ with $12 \mathrm{~cm}$ long, $0.7 \mathrm{~mm}$ wide, and $\sim 20 \mu \mathrm{m}$ thick was employed in this experiment. Different kinds of heat treatments can be programmed in the user interface. In the present case, we perform a continuous series of short heat treatment intervals $\Delta \mathrm{HT} t=45 \mathrm{~s}$, with a constant increment of the current annealing (heating scan) in the rate $\Delta I_{\mathrm{ann}} / \Delta t=10 \mathrm{~mA} / 15 \mathrm{~s}$ (shaded region in Fig. 3). During the last $\Delta t=15 \mathrm{~s}$ (enough time to let the temperature

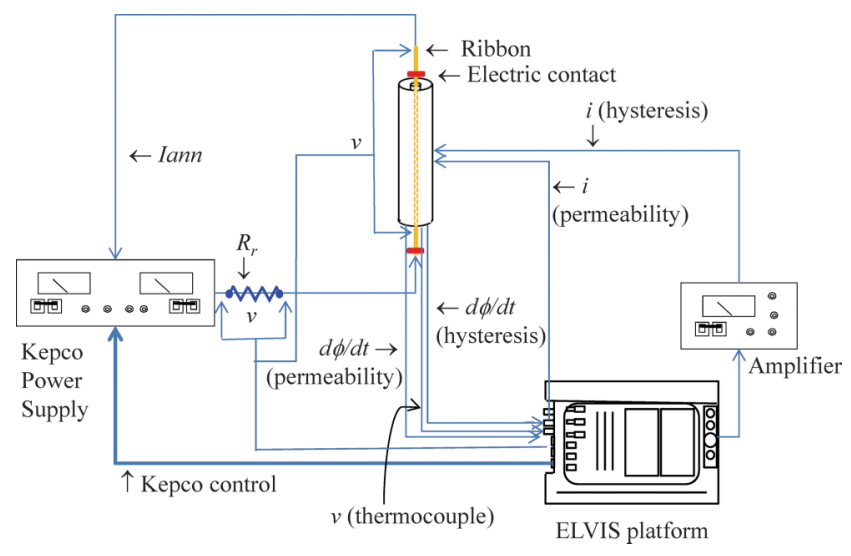

FIG. 2. Schema of experiment set-up. $I_{\mathrm{ann}}$, DC annealing current; $i, \mathrm{AC}$ current; $d \phi / d t$, magnetic flux time variation; $v$, voltage; $R_{\mathrm{r}}$, resistance.

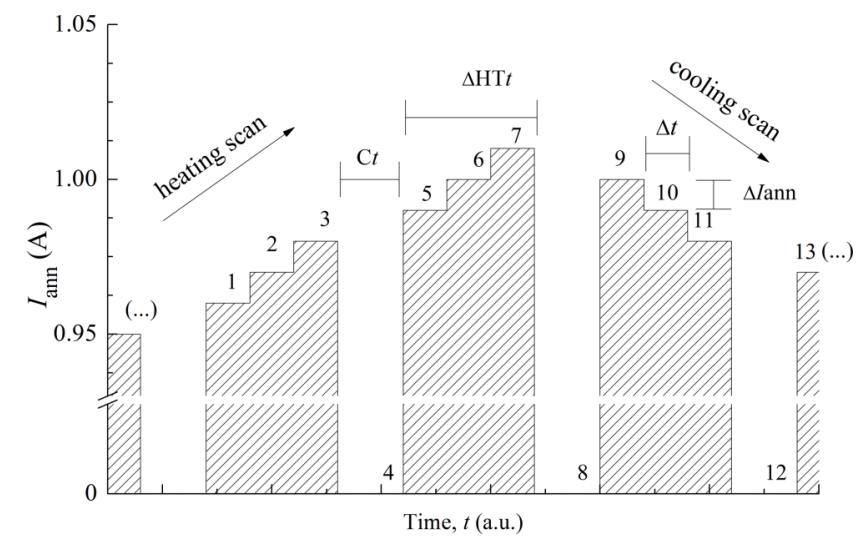

FIG. 3. Annealing current $v s$. time heat treatments. Only four steps are shown.

stabilize), the electrical resistance, the low and the high frequency magnetic characteristics of the sample in temperature are obtained consecutively. When the $\Delta \mathrm{HT} t$ is finished, the $I_{\text {ann }}$ is set to zero, and a cooling process up to room temperature for the sample immediately starts assisted by air injected to the system (blower). After certain cooling time, $\mathrm{C} t<60 \mathrm{~s}$, when the system already reaches the room temperature, the same properties above mentioned are measured again. This procedure is repeated until the programmed $I_{\mathrm{ann}}=1.010 \mathrm{~A}$ is achieved, and then the cycle is completed by returning back to the starting current (cooling scan) using the same methodology. In Fig. 3, four steps of annealing current vs. time heat treatments are schematized. The numbers indicate the time evolution of the heat treatment: at time numbered 3,7 , and 11 properties are measured in temperature (T-property) while at 4, 8, and 12 properties are measured at room temperature (RTproperty). Examples of the quantities $\Delta \mathrm{HT} t, \mathrm{C} t, \Delta t$, and $\Delta I_{\mathrm{ann}}$ are also indicated in the same Fig., as well as the heating and the cooling scan cycles. The overall duration of heat treatments was about 3 hours. Magnetic characteristics at low frequency were carried out at $50 \mathrm{~Hz}$ and under a maximum applied field $H=1.5 \mathrm{kA} / \mathrm{m}$. From the hysteresis loops we obtained the coercive fields, RT- $H_{\mathrm{c}}$ and T- $H_{\mathrm{c}}$, at room and at high temperature, respectively, and the saturation magnetizations, RT- $M_{\mathrm{s}}$ and T- $M_{\mathrm{s}}$, at room and at high temperature, respectively. Likewise, relative magnetic permeabilities, RT- $\mu_{\mathrm{r}}$ and T- $\mu_{\mathrm{r}}$, were recorded at $100 \mathrm{kHz}$ with an applied magnetic field of $0.6 \mathrm{~A} / \mathrm{m}$.

\section{RESULTS}

The results obtained from all measured properties with the JH3S are shown in Fig. 4. We can divide the structural evolution into five steps, three for the heating scan and two for the cooling scan.

\section{A. Heating scan}

- Step I: structural relaxation. At the beginning of the heating scan, no changes are detected in the measured properties up to an annealing current value of $I_{\text {ann }}$ $=0.50 \mathrm{~A}$. The unique property that is sensitive to 


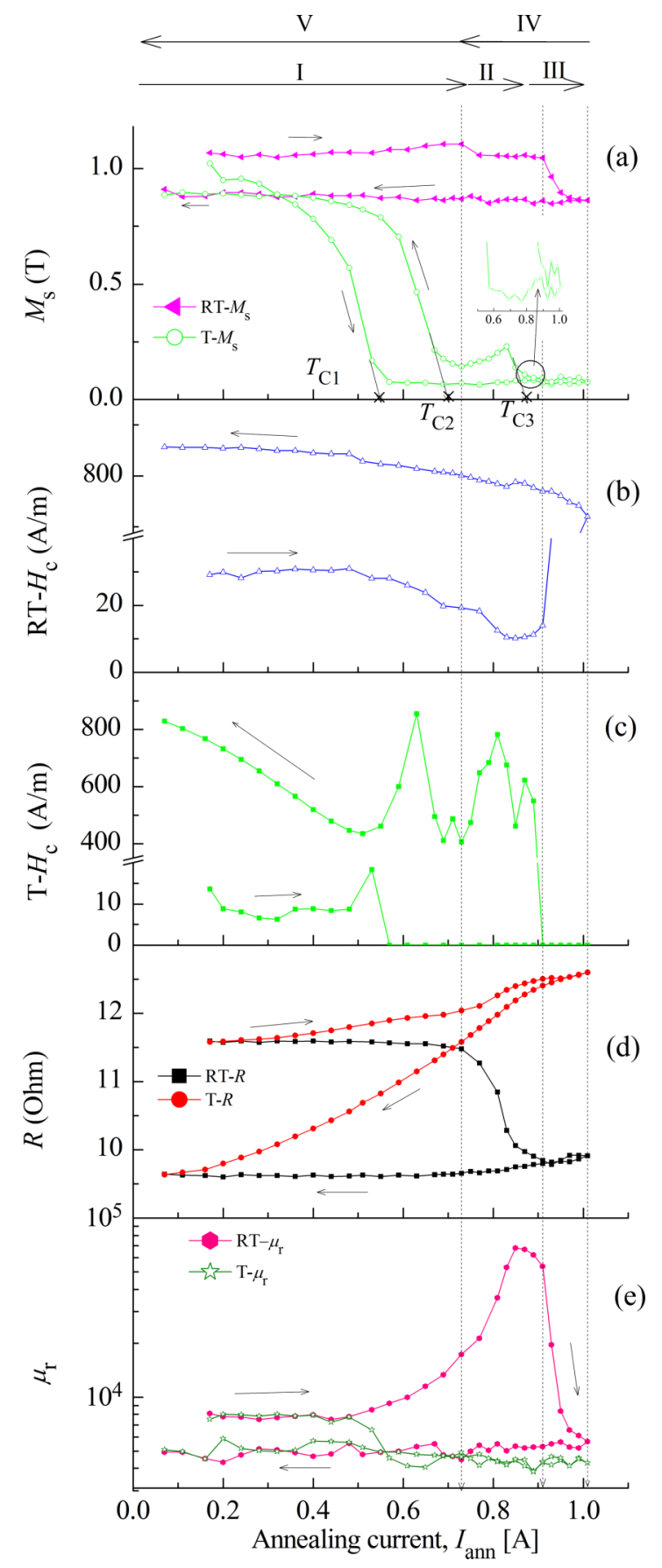

FIG. 4. All measured properties obtained simultaneously on one sample on a nanocrystalline composition alloy at high and at room temperature: (a) saturation magnetization, $M_{\mathrm{s}}$; (b) and (c) coercive field $(50 \mathrm{~Hz}), H_{\mathrm{c}}$; (d) magnetic relative permeability $(100 \mathrm{kHz}), \mu_{\mathrm{r}}$; and (e) electric resistance, $R$.

temperature in this step is the saturation magnetization, T- $M_{\mathrm{s}}$ (Fig. 3(a)). From $I_{\text {ann }}=0.50$ to $\approx 0.70 \mathrm{~A}$, a structural relaxation (stress relief) takes place and is evidenced by the room temperature properties of magnetic permeability, RT- $\mu_{\mathrm{r}}$ (increasing its value) and coercive field, RT- $H_{\mathrm{c}}$ (decreasing its value). The other RT-properties are not sensitive to this relaxation. At $I_{\text {ann }}=0.57 \mathrm{~A}$, the amorphous structure reaches its Curie temperature at $T_{\mathrm{c}}=318^{\circ} \mathrm{C}\left(\right.$ as example $\left.{ }^{4}\right)$, and the magnetic properties at temperature, T- $M_{\mathrm{s}}, \mathrm{T}-H_{\mathrm{c}}$, and T$\mu_{\mathrm{r}}$, decrease to their minimum values.
- Step II: nanocrystallization. At $I_{\mathrm{ann}} \approx 0.73$ A the nanocrystallization process starts, being supported by the irreversible changes observed in the electric resistance at room temperature, RT- $R$, and by the change in the slope of the T- $R$ curve, and it finishes at approx. $I_{\text {ann }}=0.91 \mathrm{~A}$. In this step, the soft magnetic properties reach their optimum values, at $I_{\mathrm{ann}}=0.85 \mathrm{~A}$, in RT- $H_{\mathrm{c}}$ and RT- $\mu_{\mathrm{r}}$. With respect to the behavior of the RT- $M_{\mathrm{s}}$, the high $M_{\mathrm{s}}$ of the nanocrystals $\left(M_{\mathrm{s}}=1.4 \mathrm{~T}\right.$ for $\alpha-\mathrm{Fe}(\mathrm{Si})$ with about 21 at.\% of $\left.\mathrm{Si}^{5}\right)$ is counterbalanced by the low $M_{\mathrm{s}}$ of the amorphous remnant matrix depleted in $\mathrm{Fe}\left(M_{\mathrm{s}}<0.5 \mathrm{~T}^{4}\right)$ resulting in a decrease in the overall RT- $M_{\mathrm{s}}$. Moreover, a slight increase in T$M_{\mathrm{s}}$ with a peak at $I_{\mathrm{ann}}=0.88 \mathrm{~A}$ indicates the presence of ferromagnetic nanocrystals (see inset in Fig. 4(a)) and, with increasing annealing current, at $I_{\mathrm{ann}}=0.93 \mathrm{~A}$, they reach their Curie temperature $\left(T_{\mathrm{c}}=600^{\circ} \mathrm{C}\right.$ for $\alpha$ $\mathrm{Fe}_{79} \mathrm{Si}_{21}{ }^{5}$ ).

- Step III: massive crystallization. At $I_{\mathrm{ann}}=0.93 \mathrm{soft}$ magnetic properties start to deteriorate as a consequence of the grain coarse and principally of the precipitation of Fe-borides (like $\mathrm{Fe}_{2} \mathrm{~B}, \mathrm{Fe}_{3} \mathrm{~B}$, or $\mathrm{Fe}_{23} \mathrm{~B}_{6}{ }^{6}$ ) that increase the total magnetocrystalline anisotropy of the material. This fact is reflected in a decrease in RT- $\mu_{\mathrm{r}}$ and in RT- $M_{\mathrm{s}}$ and an increase in RT- $H_{\mathrm{c}}$.

\section{B. Cooling scan}

During the cooling scans, the annealing temperature reached in a previous scan is higher than the one reached in the following scan, and so no structure transformation is supposed to occur, and the typical decreasing temperature measurements behavior is expected unless: (i) the transformation in the previous scan has not finished (not enough time to complete the transformation or the diffusion of atoms) so it will continue on the progressive scanning at a lower temperature, or (ii) the quenching produced during the cooling, $\mathrm{C} t$, period retains a high temperature structure; in this case this structure will disappear during the next successive scan.

- Step IV: The T- $M_{\mathrm{s}}$ behavior during the cooling scan presents the two-step curve typical of these materials. Two Curie temperatures can be indentified: $T_{\mathrm{c} 2}$ for $\alpha$ $\mathrm{Fe}(\mathrm{Si})$ and $T_{c 3}$ presumably for the $\mathrm{Fe}_{23} \mathrm{~B}_{6}$ compound or the $\mathrm{Fe}_{10} \mathrm{Si}_{2} \mathrm{~B}_{3}$ phase with $T_{\mathrm{c}}$ around $380-440{ }^{\circ} \mathrm{C}$, respectively. ${ }^{7,8}$ The obtained values of the $\mathrm{T}-H_{\mathrm{c}}$ in the $I_{\text {ann }}$ range from 0.91 to 0.63 A correspond to elliptical shaped not saturated hysteresis loops. This non-monotonic behavior could be related to both high temperature phases or/and magnetocrystalline changes. Also, the RT- $H_{\mathrm{c}}$ and RT- $R$ curves slightly vary perhaps as an indicative of some minor structural changes.

- Step V: From $I_{\mathrm{ann}}=0.63 \mathrm{~A}$ down to $0.07 \mathrm{~A}$, the T$H_{\mathrm{c}}$ curve presents a clear minimum at $I_{\mathrm{ann}}=0.51 \mathrm{~A}$ associated to the cancellation of the magnetocrystalline anisotropy of the phase $\mathrm{Fe}_{2} \mathrm{~B}$ that takes place at temperatures about $220{ }^{\circ} \mathrm{C} .{ }^{4} \mathrm{RT}-H_{\mathrm{c}}$ curve presents a monotonic 
and slight increase while RT- $R$ has reached its steady value. The other measured properties do not present any changes in this step, either.

\section{CONCLUSION}

With one sample, it was possible to elucidate the structural evolution by determining the presence of some phases and the thermal treatment region to optimize the magnetic properties. This was possible by the determination of several magneto-electric properties at room temperature and at high temperature. Other types of heat treatments are also possible and will be presented in the future. The system foresees the application of mechanical stresses (see Fig. 1) or the application of magnetic fields (e.g., by using Helmholtz coils) during heat treatments to study the effects of induced anisotropies. It is estimated that JH3S system presents an interesting potential for the study of these materials. Improvements are also planned for the determination of other properties, including the temperature of the ribbon.

${ }^{1}$ Soft Magnetic Materials-Technologies, "Materials, devices, new developments, industry structure and global markets," 2015.

${ }^{2}$ P. Allia, P. Tiberto, M. Baricco, and F. Vinai, Rev. Sci. Instrum. 64, 1053 (1993).

${ }^{3}$ J. A. Moya, "Low-cost fully functional virtualized instruments system for soft magnetic properties characterization" (unpublished).

${ }^{4}$ G. Herzer, in Handbook of Magnetic Materials, edited by K. H. J. Buschow (Elsevier, Amsterdam, 1997), p. 415.

${ }^{5} \mathrm{G}$. Bertotti and F. Fiorillo, "Magnetic alloys for technical applications," in Soft Magnetic Alloys Invar Elinvar Alloys, edited by H. P. J. Wijn (SpringerVerlag, Berlin, Heidelberg, 1994), p. 49.

${ }^{6}$ A. K. Panda, B. Ravikumar, S. Basu, and A. Mitra, J. Magn. Magn. Mater. 260, 70 (2003).

${ }^{7}$ H. Okumura, D. Laughlin, and M. McHenry, J. Magn. Magn. Mater. 267, 347 (2003).

${ }^{8}$ B. Idzikowski, in Properties Applications of Nanocrystalline Alloys from Amorphous Precursors, edited by B. Idzikowski, P. Švec, and M. Miglierini (Springer-Verlag, Berlin, Heidelberg, 2005), pp. 177-188. 